\title{
Mentality Differences in the Field of Medicine: Challenges in Cross-Cultural Collaborations in Austrian and German Hospitals between Native Physicians and Immigrated Colleagues from Poland and Hungary
}

Mario Rubenzer

\author{
Fachhochschule Oberösterreich, Hagenberg, Austria
}

\begin{abstract}
Within the last decades, studies about characteristics of national culture have mostly been focused on the business sector. In other industries, like the field of medicine, research about mentality differences is still in its infancy. Due to increased international job mobility of physicians around the globe, especially within the European Union, it is considered necessary to draw more attention at this topic. For this purpose, some studies are presented initially that have already addressed the issue of cultural differences in the field of medicine. By extracting their most crucial findings, an overview regarding the state-of-the- art is provided. Based on these findings, a design for qualitative research is modelled that is aimed at closing these knowledge gaps. As a first step in this context, it is explained which perspective is considered to be the most promising in terms of generating in-depth insights. Moreover, a possible approach towards constructing mentalities beyond a national level is introduced. This part goes hand in hand with suggesting two mentalities whose comparison is regarded to make sense. These mentalities are going to be an Austro-Germanic Mentality (physicians from Austria and Germany) and a Magyar- Slavic Mentality (physicians from Hungary and Poland). Subsequently, the author is going to recommend some dimensions of established cultural models that should be taken into account when designing an interview outline. Beyond that, some preliminary findings of an ongoing study with Eastern European doctors who are working in Austrian and German hospitals are presented. So far, it has become obvious that there is a remarkable different approach towards hierarchy and leadership, personal relationships with colleagues as well as task-fulfillment and personal responsibility. In the last part of this paper, the author emphasizes the positive effects that are expected to occur when awareness in terms of mentality differences is raised and how they could be achieved.
\end{abstract}

Keywords: Mentality Differences, Medicine: Challenges, Cross-Cultural Collaboration, Immigration

\section{Introduction}

Differences in national cultures have been examined widely in the context of global business operations (Trompenaars \& Hampden-Turner, 1998) and international projects (Popescu et al. 2014). They are, however, not only a relevant issue for building and maintaining economic relationships between countries. While regarding knowledge in terms of cultural differences as important when it comes to making deals and money, little scientific attention seems to be drawn at cultural aspects as a relevant factor of retaining valuable human resources, for example in the field of medicine. Despite the fact that the "last decade has shown a growing interest in cross-national studies in medical communication in European health care, (...) studies which 
Mentality Differences in the Field of Medicine: Challenges in Cross-Cultural Collaborations in Austrian and German Hospitals Between Native Physicians and Immigrated Colleagues from Poland and Hungary

Mario Rubenzer

incorporate cultural dimensions of nations are mostly lacking" (Meeuwesen et al. 2009, p. 58). Mentality differences, however, do not only affect explicit communication, as they occur in every sphere of the daily job life of physicians working abroad. National culture has an undeniable impact on the way doctors interact with colleagues and patients as well as on the self-conception of their role and on how they deal with certain events and situations.

From the viewpoint of countries attracting foreign medical doctors, cultural diversity offers both chances and risks. Thus, it is in the best interest of hospitals around the globe to increase their knowledge concerning mentality differences. Identifying, extracting and interpreting cultural differences is challenging, however. While differences in national culture have been wellresearched within the last decades in the context of business - leading to a variety of tools and useful information available for managers - research on this issue in the field of medicine is still at the beginning. As global job mobility of high-skilled workers, especially physicians and other health personnel, keeps increasing, it is considered as essential to raise awareness for this topic, though.

\section{Mentality Differences in Medicine: State-of- the-Art}

Most of the quite sparse literature on this issue only provides superficial insights, solely addresses particular aspects or is questionable in terms of representativeness because of its small sample size. Nonetheless, the findings generated so far serve as an important starting point for further research on this topic. Within the sections below, some of the most flamboyant aspects in current research concerning mentality differences in the field of medicine are identified, briefly discussed and interpreted by referring to dimensions included in some of the most established models concerned with national culture.

\subsection{Differences in Communication}

Despite its narrow focus, the first study that needs to be mentioned in this context is the one of Skjeggestad et al. (2017). The scientists found out that all of their participants "spontaneously described language barriers as the most prevalent problem in their everyday collaborations" ( $p$. 1468). This insight itself is little of a surprise, as being able to speak and understand a foreign language when working abroad is essential for doctors if they want to be successful in their profession. Interesting, however, are the factors this central finding is built upon and the further insights derived from it:

"The language barriers participants described do not have clear associations with the IMGs' [international medical graduates'] nationality or position. This illustrates that IMGs are individuals, and language barriers are contextual and multifaceted, involving aspects that are more complex than language issues alone. Indeed, an interesting finding in our study is that what participants described as merely language barriers actually included a range of topics that included semantics, pragmatics, and cultural aspects of language use (...)" (p. 1470).

Referring to the cultural aspects addressed, Skjeggestad et al. interpreted their insights as "culture related patterns of communication in particular missed in sensitive situations (e.g., around death, sexual health and orientation)" (p. 1469). As an example, they used the statement of a Norwegian physician. The doctor once saw that one of his foreign colleagues did not show empathy in a way natives would have in a situation where relatives were surrounding the bed of a recently deceased person:

"He comes into the room, he pulls out the medical chart, turned on his heel and left the room without any communication with the relatives" (p. 1469). What was abstained from in context of 
Mentality Differences in the Field of Medicine: Challenges in Cross-Cultural Collaborations in Austrian and German Hospitals Between Native Physicians and Immigrated Colleagues from Poland and Hungary

Mario Rubenzer

the study, was trying to explain this kind of behavior. From the author's point of view, there are different ways of interpreting this different behavior, depending on the cultural model referred to. From Hall's (1976) perspective, the situation might depict differences in low- and high-context communication. In a high-context country, for example, remaining silent in a situation like the one described above might be a way of expressing respect. In a low-context country like Norway, however, sympathy might always have to be conveyed verbally as it is the only way to do so.

\subsection{Differences in Norms and Values}

On the other hand, this kind of (mis)behavior described by Skjeggestad and colleagues could be due to different social norms: In a rule-oriented, polite but direct country that Lewis (2006) considers as linear-active, for example, proactively offering one's condolence to the bereaved might be considered as compulsory. In countries Lewis regards as reactive, however, which are known for being rather restrained and defensive, breaking the silence in a moment of grief might not be considered as tactful.

In another study, Kolodziej (2016) conducted a quantitative survey with 32 Polish doctors who were working in Germany. Because of her small sample size, her findings can only be regarded as indications. Among these indications, however, an aspect is found that can be related to cultural differences: More than 30 per cent of the study's participants stated that they felt let down by their colleagues as they did not provide the kind of support they were hoping for. Because of their disappointment, these participants said that they would rather recommend young medical doctors to stay in Poland than to come to Germany. The perceived lack of support can be interpreted by referring to Lewis' model: As Poles are considered as multi- active, i.e. rather emotional and people-oriented, they might tend to show more empathy for their colleagues and support struggling co-workers proactively because of an intrinsic motivation. Germans, however, are rated as linear-active. That means they merely concentrate on their own tasks, do not seek to strengthen private ties to their colleagues and only provide help when they are explicitly asked for it. The behavior could be explained by referring to other dimensions, too, for example differences in ingroup-collectivism (GLOBE, n.d.): The cohesiveness within Polish groups appears to be stronger, which may result in a higher probability that their members will help each other.

Cultural differences were also identified in the study of Legido-Quigley et al. (2015). The researchers interviewed 23 immigrated physicians serving in the United Kingdom concerning their experiences with the British NHS (National Health Service) and perceived differences to the health care system in their EU country of origin. Even though cultural issues where not addressed explicitly in their narrative interviews, some aspects regarding this topic were referred to proactively by the participants: Some of them noticed differences in power distance (GLOBE, n.d.), telling that they regarded the British NHS as "less hierarchical, particularly with the way in which consultants work and how much responsibility interviewees mentioned having as junior doctors" (Legido-Quigley et al. 2015, p. 498). A further explanation of this discovery revealed that this fact was seen as ambiguous: positive in terms of shown trust, but negative because of unclear responsibilities.

\section{Developing a Research Design for Mentality Differences in Medicine}

The findings stated in chapter 2 show two shortfalls of current research on mentality differences in the field of medicine: Firstly, only a few situations and areas in which cultural misunderstandings are likely to occur have been addressed so far. Secondly, the existing insights need to be further reviewed in order to detect the underlying reasons. Even though scientists acknowledge that some differences in communicational and behavioral patterns of native and foreign doctors might be related to culture, they seldom provide an in-depth- examination of 
Mentality Differences in the Field of Medicine: Challenges in Cross-Cultural Collaborations in Austrian and German Hospitals Between Native Physicians and Immigrated Colleagues from Poland and Hungary

Mario Rubenzer

this issue in most cases. Therefore, the author's interpretations of the existing insights are rather speculative. In order to change that, prospective studies concerning mentality differences in the field of medicine should focus on identifying further situations and events in which cultural differences become visible. This effort should be combined with an attempt to clarify the reason for these differences by referring to dimensions of established cultural models. To achieve this goal, however, it is necessary to prepare a useful research design first. This is done in seven steps:

- Defining the perspective mentality differences are looked at from

- Deciding on the mentalities to compare

- Detecting specifics of a hospital physician's work routine

- Determining cultural dimensions to address

- Developing an interview outline based on these criteria

- Discussing and interpreting insights derived from the interviews

- Drawing conclusions in order to make practical implications

The first four of them are going to be explained on the following pages, followed by a presentation of preliminary findings gained in context of a yet ongoing study.

\subsection{Defining the Perspective}

As a first step towards this direction, Trompenaars and Hampden-Turner (1998) advise people confronted with culture-related issues to develop a better understanding of "their own culture and cultural differences in general, by learning how to recognise and cope with these" (p. 2). Self-assessment is quite difficult, however, as it is self-evident that critically reflecting the culture people were born and raised in is impossible without having a reference culture to compare it to. In the following sections, it is explained why immigrants rather have an advantage when it comes to developing cultural awareness.

\subsubsection{Cognitive Hurdles of Natives}

When asked to explain their own culture, people are often seen to be limited to describing superficialities and artefacts like food, dress codes and art that Trompenaars and HampdenTurner (1998) call "observable reality" (p. 21). There is a second cognitive hurdle, however, which regards assessing foreign cultures: Physicians who have never lived abroad for a longer period of time are quite likely to have what Bennett (2004) calls an "ethnocentric world view" (p. 1). According to him, this kind of world view can be divided in three degrees of cultural ignorance, which are Denial, Defense and Minimization, with the stage of ethnocentrism varying from country to country. A Denial world view is seen as characteristic for Germans, for example. Bennett (2004) describes this stage as follows:

"This is the state in which one's own culture is experienced as the only real one - that is, that the patterns of beliefs, behaviors, and values that constitute a culture are experienced as unquestionably real or true. (...) As a result, cultural difference is either not experienced at all, or it is experienced as associated with a kind of undifferentiated other such as 'foreigner' or 'immigrant'"' (p. 2).

It is not necessarily the case, however, that highly educated members of a society - in this case physicians - show the same tendency as the overall population. An indication that this assumption might be correct, though, can be found in the study of Legido-Quigley et al. (2015): Some of their study's participants, all of them doctors originating from EU countries, reported that they saw themselves confronted with "'stereotyping' and 'patronising behaviour'" (p. 499) in the United Kingdom. This finding implies that British physicians tend to be at the second level of ethnocentrism called Defense. This stage is characterized by members of a society believing 
Mentality Differences in the Field of Medicine: Challenges in Cross-Cultural Collaborations in Austrian and German Hospitals Between Native Physicians and Immigrated Colleagues from Poland and Hungary

Mario Rubenzer

that they are part of "the most 'evolved' form of civilization" (Bennett 2004, p. 3), regarding their own culture as superior and others as inferior. Thus, chances are low that asking doctors who have never lived abroad about their observations of cultural differences lead to in-depth insights about cultural differences, as they might not be able to notice them at all.

\subsubsection{Biculturalism of Immigrants}

Keeping such preconditions in mind, it becomes obvious that in order to get a realistic impression of cultural differences in the field of medicine, it is necessary to look at one's own culture through the eyes of strangers. Or to be more precise: To conduct qualitative interviews with immigrated doctors who perceive these mentality differences on a daily basis. In order to succeed abroad, foreign physicians are forced to deal with and adopt cultural characteristics "as a result of some social influences and of the cultural environment pressure" (Cormos 2014, p. 242). Dau (2016) regards this development as becoming bicultural, a stage where immigrated "individuals have internalized two cultural frameworks" (p. 49). Reaching such a state of mind, however, enables foreign doctors to identify, critically reflect and name differences in terms of mentality. Therefore, interviewing them is more likely to reveal differences in mentality that lie beyond the level of visible artifacts.

\subsection{Deciding on Mentalities to Compare}

When it comes to creating a research design, it is impossible to compare all countries to each other in terms of cultural differences. Because of that, a selection has to be made beforehand. In this process, it has to be decided on both a host mentality (members of a native society being assessed) and a guest mentality (immigrants of a foreign society reviewing the host culture). This restriction to a host and a guest mentality does not necessarily have to be made on a country level: In order to get general insights that are applicable for a bigger society, it seems legitimate to compare two sets of countries to each other that have proven to be similar in terms of national culture. The author is well aware that each country differs from another one to some extent, but so do individuals originating from these countries. There are, however, some countries which have a lot in common and whose national cultures can be combined under a broader term of mentality. This claim is argued in the sections below:

Taking a look at Lewis' cultural triangle (fig. 1) leads to a first clue: Therein, Poland and Hungary (multi-active) as well as Austria and Germany (linear-active) can be found in the same cultural spectrum. Each of these culture types is considered to possess similar cultural characteristics, both on the cultural level of the observable and tangible (intensity of talking, use of body language) and on the level of norms and values (displaying feelings, amount of planning etc.).

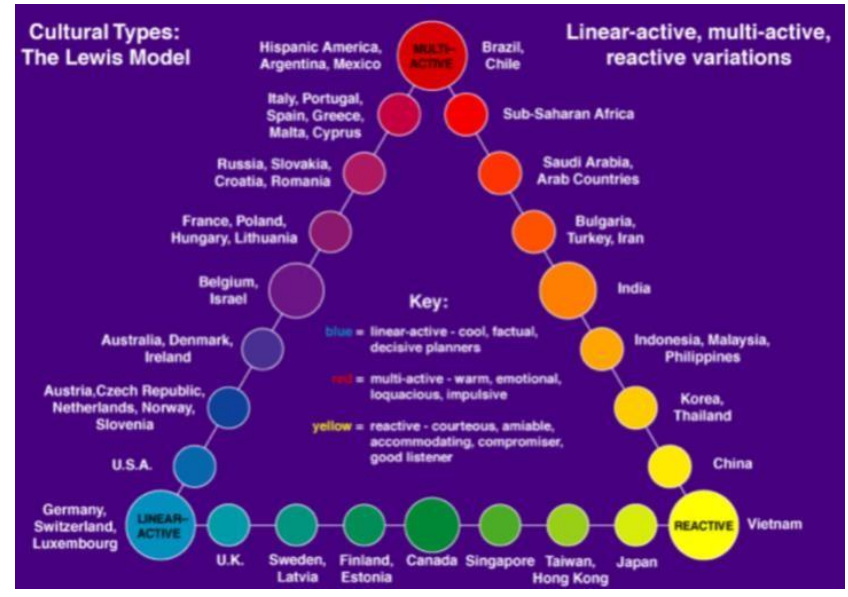

Figure 1: Assignment of cultures based on Lewis' model (Cross-Culture 2015) 
Mentality Differences in the Field of Medicine: Challenges in Cross-Cultural Collaborations in Austrian and German Hospitals Between Native Physicians and Immigrated Colleagues from Poland and Hungary

Mario Rubenzer

This impression seems to be confirmed by the findings of Bakacsi et al. (2002) and Szabo et al. (2002). In an effort to group countries with a similar mentality, the scientists created cultural clusters based on the findings of the GLOBE study. In their findings, both Szabo and colleagues and Bakacsi and colleagues differentiated between "Should be" values and "As is" practices of the countries. These two scores differed remarkably. Within the following sections, only the scores of the actual practices of countries are looked at in detail, as they are, in contrast to values, visible for foreigners in daily work life.

The first of the two groups is the Eastern European cluster, in which Poland and Hungary are included. In terms of actual practices, these countries show remarkable similarities in most of the GLOBE's nine cultural dimensions (table 1). Only in two dimensions - institutional collectivism and assertiveness - the Polish and Hungarian means differed from one another by more than 0.5 points. When looking at the Germanic European cluster, the same can be considered true for Austria and Germany. What has to be mentioned at this point is that in their work, Szabo et al. (2002) divided Germany - due to its dispartment back in the days of Cold War - into a former Western and a former Eastern part. In all dimensions, West Germany and East Germany scored very similar (less than 0.5 points difference) to almost identical means. 17 In order to be able to directly compare Germany to Austria, the author had to aggregate the scores of the two German parts to an overall score. The comparison showed, with only a few exceptions, remarkable similarities between Austria and Germany in terms of practices (table 1).

The fact that two established cultural models with fundamental different approaches towards assessing culture come to the same conclusion, however, implies that merging them under a broader term of mentality is legitimate. Therefore, Austrian and German doctors are regarded as representatives of an Austro-Germanic mentality (AGM), whereas Hungarian and Polish doctors are considered as representatives of a Magyar-Slavic mentality (MSM).

Table 1: Comparison of GLOBE study findings regarding Austria, Germany, Hungary and Poland extracted from works about the Germanic (Szabo et al. 2002) and the Eastern European cluster (Bakacsi et al. 2002). A score of $<3,5$ is considered low, a score between 3,5 and 4,50 is considered medium and a score that is $>4,50$ is considered high.

\begin{tabular}{|l|l|l|l|l|l|l|}
\hline \multirow{2}{*}{\multicolumn{1}{|c|}{ GLOBE dimensions }} & \multicolumn{5}{|c|}{ practices (mean score) } \\
\cline { 2 - 7 } & Austria & Germany & AGM Av. & Hungary & Poland & MSM Av. \\
\hline Uncertainty Avoidance & 5,16 & 5,19 & 5,18 & 3,12 & 3,62 & 3,37 \\
\hline Future Orientation & 4,46 & 4,11 & 4,29 & 3,21 & 3,11 & 3,16 \\
\hline Power Distance & 4,95 & 5,40 & 5,18 & 5,56 & 5,10 & 5,33 \\
\hline Institutional Collectivism & 4,30 & 3,68 & 3,99 & 3,53 & 4,53 & 4,03 \\
\hline Humane Orientation & 3,72 & 3,29 & 3,51 & 3,35 & 3,61 & 3,48 \\
\hline Performance Orientation & 4,44 & 4,17 & 4,31 & 3,43 & 3,89 & 3,66 \\
\hline Group \& Family Collectivism & 4,85 & 4,27 & 4,56 & 5,25 & 5,52 & 5,39 \\
\hline Gender Egalitarianism & 3,09 & 3,08 & 3,09 & 4,08 & 4,02 & 4,05 \\
\hline Assertiveness & 4,62 & 4,64 & 4,63 & 3,23 & 3,75 & 3,49 \\
\hline
\end{tabular}

Note: As Germany was splitted in Former Western and Former Eastern Germany in context of the GLOBE Study, the German score is a calculated average in a ratio $1: 1$.

Now that this determination is made, it remains to sort out whether it makes sense to compare these two mentalities to one another. There are two reasons why this question can be answered with yes: First of all, as stated above (see chapter 3.1.1), Germany (respectively Austria) is rather associated with having an ethnocentric world view in the stage of Denial. This stage is associated with a cognitive conditioning that lacks the capability to distinguish between cultures. 
Mentality Differences in the Field of Medicine: Challenges in Cross-Cultural Collaborations in Austrian and German Hospitals Between Native Physicians and Immigrated Colleagues from Poland and Hungary

Mario Rubenzer

Thus, physicians originating from these countries are likely to need more professional assistance when it comes to developing awareness for and an understanding of mentality differences. Not only the native physicians would benefit from research on this topic, though: Kolodziej (2016) found out that almost all of the Polish doctors she interviewed in her study would have appreciated "integration and language lessons in context of the integration process" (p. 69) in the first month of their stay abroad. Contributing to a better understanding between doctors with AGM and MSM does not only make sense from a social, but also from an economical point of view: Both Germany and Austria are, due to their wealth, a popular destination for Eastern European doctors. As the statistics of the Bundesärztekammer (2016) reveal, around $11 \%$ of all physicians practicing in Germany are immigrants, with approximately every tenth foreign doctor originating from either Poland or Hungary. The same is true for Austria: According to the MedienServicestelle Neue Österreicher/innen (2016), 1.2\% of all foreign medical doctors working in Austria in 2016 were born and raised in either Poland or Hungary. Hungarian physicians (366) even form the biggest group of non-German-speaking foreign doctors. As representatives of MSM account for a remarkable part of all foreign doctors in both Austria and Germany examining their differences in mentality seems legitimate.

\subsection{Detecting Specifics of a Hospital Physician's Work Routine}

Designing a purposeful interview outline requires detailed knowledge of the daily work routine of a hospital doctor. It is necessary to be aware of the tasks, processes, situations, events and people physicians are involved in or confronted with in order to assess which questions to ask in what context. Being able to address issues that doctors can easily relate to increases the probability of receiving answers that grant insight in cultural differences that reaches beyond the surface. By making foreign physicians rather tell stories about strange situations they have encountered than asking them for explicit observations, chances are high that the information provided contains hints that are hidden in between the lines. Therefore, an interview with a medical doctor has to be conducted before the actual research starts in order to obtain general knowledge concerning his or her job routine.

\subsection{Determining Cultural Dimensions to Address}

Letting medical doctors narrate their experiences might be the right approach when it comes to gleaning aspects that have not been thought of in the development process of the interview outline. That does not mean, however, that proactively addressing cultural dimensions can be abstained from completely. Without profound preparation, it can happen that interviewees switch their narrative focus and refer to issues that cannot be related to mentality primarily or at all. In order to avoid that, it is necessary to decide on a set of dimensions that has proved to be effective in terms of examining and interpreting national culture. This set should be taken into account when designing the interview outline. From the author's viewpoint, two established cultural models need to be considered as they complement one another: The first one is the GLOBE (n. d.), which divided national culture into a set of nine dimensions:

- Performance Orientation;

- Assertiveness;

- Future Orientation;

- Humane Orientation;

- Institutional Collectivism;

- In-Group Collectivism;

- Gender Egalitarianism;

- Power Distance;

- Uncertainty Avoidance; 
Mentality Differences in the Field of Medicine: Challenges in Cross-Cultural Collaborations in Austrian and German Hospitals Between Native Physicians and Immigrated Colleagues from Poland and Hungary

Mario Rubenzer

The GLOBE (n. d.) is interesting for two reasons: Firstly, aside of own aspects (e.g. Gender Egalitarianism9), it includes dimensions - or at least selected parts of them - that can be found in other established cultural models (e. g. Hofstede, Trompenaars \& Hampden-Turner). Secondly, the GLOBE study not only examined cultural differences in terms of social norms and values, but also in terms of actual practices. In contrast to values, which are located in a person's mind and are therefore not visible to the eye, practices are something that an attentive person is able to spot and to interpret. Because of that, the author assumes that differences in practices between AGM and MSM might not only be recognizable in the context of business, but also in the field of medicine. It is clear that not all of the GLOBE's dimensions can be reviewed in detail. Therefore, a selection of the most relevant ones has to be made beforehand. The second cultural model that needs to be taken into account is the one of Lewis, as it contains important aspects that are not addressed in the GLOBE study at all. The items Lewis used to distinct between linear-active, multi-active and reactive countries can mostly be related to three factors: time, degree of emotional control and unwritten laws of communication. These aspects can be found in other established cultural models, too: A detailed description and discussion of unwritten laws of communication was provided by Hall (1976), for instance, who coined the terms low-context (high level of explicitness) and high-context (low level of explicitness) communication. Trompenaars and Hampden-Turner (1998) concerned themselves with an in- depth examination of the culture-dependent extent of emotional self-control. Based on their findings, they introduced the dimension neutral vs. emotional. Differences in the perception of and dealing with time have also proved to be a relevant issue in the past: Hall and Hall (1990) distinguished between monochronic people (preference for making plans and doing one thing after another) and polychronic people (preference for multi-tasking and improvising). As Lewis inherited some of the most important characteristics of these three dimensions in his cultural triangle, his model is regarded as a useful supplement of the GLOBE study dimensions.

\section{Preliminary Findings}

In order to find out which mentality differences become the most obvious in the daily work of doctors, some exploratory field work is undertaken. Even though the sample of interviewed physicians from Poland and Hungary taken into account so far is quite small, it is possible to carve out some consistent characteristics. As the study has not been completed yet, only a superficial review and interpretation of the insights gathered so far can be provided at this point. Thus, despite seeming to be relatable to some established dimensions of the cultural models mentioned beforehand, another terminology is used in order to topically group the current findings. They are described in detail in the following paragraphs:

\subsection{Hierarchy and Leadership}

When it comes to hierarchy, Polish and Hungarian physicians tend to have another conception of how bosses respectively supervisors should behave towards and should be treated by subordinates. In the MSM, the hierarchical system was compared to the structure "in the military". This means that a chief physician in MSM is rather used (and expected) to make decisions and give orders, with his subordinates executing his or her will and reporting him or her all the information he or she is seeking for. Other persons that occupy higher positions in the formal hierarchy are also considered to be powerful enough to tell younger, lesser experienced colleagues what to do and when to do it. In an MSM society, questioning, criticizing or disagreeing with superior physicians commonly leads to severe consequences, starting from getting shouted to humiliation and threats or may even result in a loss of the job. In the AGM, the chief physician rather holds the function of an adviser. Instead of only being concerned with his or her own issues, a chief physician in Austria and Germany is more of a team member that accompanies his or her subordinates and who is closely involved in their daily work routine. His or her opinion on what to do and how to do it is rather perceived as a recommendation than an 
Mentality Differences in the Field of Medicine: Challenges in Cross-Cultural Collaborations in Austrian and German Hospitals Between Native Physicians and Immigrated Colleagues from Poland and Hungary

Mario Rubenzer

order. Furthermore, younger doctors in Austria and Germany are not punished for sharing divergent thoughts and their ideas are likely to be taken more serious in discussions. Another finding is that the way nurses and orderlies in Austria and Germany treat doctors differs from the way that is typical in Poland or Hungary. Health personnel with AGM is considered to be much more "independent" from doctors, which means its representatives are more likely to refuse to take an order or answer back whenever they disagree with a decision. Moreover, they seem to use a more informal, familiar tonality when talking to doctors, which is rather perceived as a lack of respect by physicians from Poland and Hungary.

\subsection{Personal Relationships between Colleagues}

Another big difference in terms of mentality can be found in the intersection of the professional and the private sphere: Doctors with an AGM tend to separate their work from their private life. This means that they do not seek to strengthen personal ties between co-workers and avoid dealing with any job-related issues in their free time. Physicians with an MSM, on the other hand, have a completely different approach. They tend to see their colleagues either as friends or foes, but not neutrally as just coworkers. Because of that, establishing a personal relationship to colleagues is rather the rule than the exception in Poland and Hungary. Furthermore, work is not restricted to the hours spent in the hospital: Whenever an issue related to works comes in their mind, representatives of the MSM contact each other to discuss this topic right away. This kind of behavior was hardly ever noticed in Austria/Germany.

\subsection{Task-Fulfillment and Personal Responsibility}

Some of the interviewees from Eastern Europe explicitly addressed a difference in mentality when it comes to organizing work. They considered it rather ineffective that the daily work life of doctors and health personnel in Austria and Germany is dominated by schedules, plans and checklists. This mindset of sticking to predefined tasks and timelines is seen to prevent collaboration to some extent, as some goals of nurses and doctors in the hospital appear to be "parallel and are never going to meet". Furthermore, some physicians with MSM considered that the personal responsibility of doctors with AGM is lower than in Poland or Hungary. Being tied to plans and schedules was regarded to sometimes keep them from doing effective work and re-setting priorities whenever a situation requires such an adaptive action. Moreover, doctors with MSM perceive to be limited in their professional discretion and liability towards patients by getting detailed instructions on what to do and when to do it.

\section{Conclusion}

In order to be able to reduce or avoid negative effects or feelings resulting from misconceptions and different internalized standards, hospitals need profound knowledge of their (native and foreign) employees' cultural background and the characteristics related to them. Based on these findings, the human resources departments can develop purposeful workshops for native physicians and integration programs for immigrated doctors. As Kolodziej (2016) writes, this measure is regarded as particularly helpful if is offered in an early stage of foreign doctors' employment. In order to add a social component, cultural awareness programs for native and foreign doctors could also be combined, for example in form of mixed courses. Implementing such measures is not only likely to improve the work climate in culturally diverse hospitals and to increase the efficiency of cross-cultural team work in the field of medicine: A further positive side effect could be the developing of more openness towards alternate approaches and new ideas, which might lead to new impulses in terms of research and innovation. Studies in the context of international projects have already conveyed an impression of the potential cultural diversity bears: "People with different cultural backgrounds, working together, may bring creativity, new ideas in the projects collaboration and new approaches to problem solving" (Popescu et al. 2014, p. 526). More cultural awareness and approximation could also result in a 
Mentality Differences in the Field of Medicine: Challenges in Cross-Cultural Collaborations in Austrian and German Hospitals Between Native Physicians and Immigrated Colleagues from Poland and Hungary

Mario Rubenzer

higher probability that immigrated doctors decide to settle down in their host country permanently. As a lot of native doctors are expected to retire within the next 15 years in Austria and Germany, the latter is a fact that the domestic health care system in both countries would benefit from.

\section{References}

- Bakacsi, Gyula, Sandor Takacs, Karacsonyi Andras, and Imrek Viktor (2002), "Eastern European Cluster: Tradition and Transition," Journal of World Business, 37, 69-80. Crossref

- Bennett, Milton J. (2004), "Becoming Interculturally Competent," in Toward Multiculturalism: A Reader in Multicultural Education, J.S. Wurzel, ed. Newton, MA: Intercultural Resource Corporation, 1-13.

- Bundesärztekammer (2016), "Ärztestatistik zum 31. Dezember 2016. Bundesgebiet gesamt," (accessed February 26, 2018), [available at http://www.bundesaerztekammer.de/fileadmin/user_upload/downloads/pdfOrdner/Statistik2016/Stat16AbbTab.pdf].

- Cormos, Viorica C. (2014), "Mentality and Change in the Context of International Migration," Procedia - Social and Behavioral Sciences, 149, 242-247. Crossref

- CrossCulture (2015), "The Lewis Model - Dimensions of Behaviour," (accessed February 26, 2018), [available at https://www.crossculture.com/latest-news/the-lewis-modeldimensions-of-behaviour/].

- Dau, Luis A. (2016), "Biculturalism, Team Performance, and Cultural-Faultline Bridges," Journal of International Management, 22, 48-62. Crossref

- Hall, Edward T. (1976), Beyond Culture. New York: Anchor Books, Doubleday.

- Hall, Edward T. and Mildred R. Hall (1990), Understanding Cultural Differences. Michigan: Intercultural Press.

- GLOBE Study (n. d.), "Understanding the Relationship between National Culture, Societal Effectiveness and Desirable Leadership Attributes: A Brief Overview of the GLOBE Project 2004," (accessed February 26, 2018), [available at http://globeproject.com/study_2004_2007].

- Kolodziej, Monika (2016), "Welchen Beitrag können in Polen ausgebildete Ärzte zur Behebung des Ärztemangels in Deutschland leisten? Vergleich von Medizinstudium und Facharztweiterbildung in Deutschland und Polen unter exemplarischer Darstellung von Innerer Medizin, Allgemeinmedizin und Anästhesie," doctoral dissertation, Medizinische Fakultät, Ludwig-Maximilians-Universität zu München.

- Legido-Quigley, Helena, Saliba, Vanessa, and McKee, Martin (2015), "Exploring the Experiences of EU Qualified Doctors Working in the United Kingdom: A Qualitative Study," Health Policy, 119, 494- 502. Crossref

- Lewis, Richard D. (2006), When Cultures Collide. Leading Across Cultures (3. ed.). Porvoo: WS Bookwell.

- Medien-Servicestelle Neue Österreicher/innen (2016), "63.600 MigrantInnen arbeiten im Gesundheitswesen," (accessed February 27, 2018), [available at http://medienservicestelle.at/migration_bewegt/2016/05/11/63-600-migrantinnenarbeiten-im- gesundheitswesen/].

- Meeuwesen, Ludwien, Atie v. d. Brink-Muinen, and Geert Hofstede (2009), "Can Dimensions of National Culture Predict Cross-National Differences in Medical Communication?" Patient Education and Counseling, 75, 58-66. Crossref

- Popescu, Anca-Diana, Borca, Cristina, Fistis, Gabriela, and Draghici, Anca (2014), "Cultural Diversity and Differences in Cross-Cultural Project Teams," Procedia Technology, 16, 525531. Crossref

- Skjeggestad, Erik, Jennifer Gerwing, and Pal Gulbrandsen (2017), "Language Barriers and Professional Identity: A Qualitative Interview Study of Newly Employed International Medical 
Mentality Differences in the Field of Medicine: Challenges in Cross-Cultural Collaborations in Austrian and German Hospitals Between Native Physicians and Immigrated Colleagues from Poland and Hungary

Mario Rubenzer

Doctors and Norwegian Colleagues," Patient Education and Counseling, 100, 1466-1472. Crossref

- Szabo, E. et al. (2002), "The Germanic Europe Cluster: Where Employees Have a Choice," Journal of World Business, 37, 55-68. Crossref

- Trompenaars, Fons and Charles Hampden-Turner (1998), Riding the Waves of Culture. Understanding Cultural Diversity in Business (2. ed.), Helsinki: Werner Söderström Oy. 